\title{
Le déclin du Port de Québec dans la deuxième moitié du XIXe siècle
}

\section{Pierre Camu}

Quebec City, the principal Canadian port since the time of the French regime, declined during the last part of the nineteenth century, particularly with respect to Montreal, which was better placed to serve as the maritime gateway to the rapidly developing continental communications of North America. There is some confusion about Quebec's situation because existing accounts treat in isolation what in fact were two areas of decline: ocean shipping traffic, and the construction of large wooden sailing vessels in the port's shipyards. The present article addresses both these developments, and also examines efforts by local political and business leaders to improve and modernize the port's facilities, measures that would contribute to increased traffic in the early part of the twentieth century.

Il y a une certaine confusion qui existe au sujet du déclin du trafic portuaire de Québec dans la deuxième moitié du XIXe siècle, parce qu'on a analysé et étudié séparément deux déclins, sans les réunir: celui du trafic océanique et celui de la construction des grands voiliers en bois dans ses chantiers maritimes. ${ }^{1}$ Les causes de ces deux revers sont différentes, mais leurs effets combinés, survenus en même temps, ont provoqué un ralentissement sérieux des activités du milieu maritime régional.

Le port de Québec avait été tour à tour, pendant 225 ans, le grand port de la Nouvelle-France (1608-1760), de la colonie britannique de Québec (1760-1794), des Bas et Haut Canada (1794-1840), et de la province unie du Canada (1841-1867), avant de perdre son titre de port national dans les années qui suivirent la création de la Confédération de $1867^{2}$ (figure 1). On parla alors de déclin quasi irréparable, d'effondrement, de perte considérable et de désintéressement du monde des affaires et

1 Deux auteurs ont utilisé le mot « déclin » pour expliquer ce qui arriva, l'un au sujet du port, l'autre au sujet de la construction navale. André Lemelin, « Le déclin du port de Québec et la reconversion économique à la fin du XIXe siècle: une évaluation de la pertinence de l'hypothèse du staple », Recherches sociographiques 22, $\mathrm{n}^{\circ} 2$ (1981), 155-186. Albert Faucher, "The decline of shipbuilding at Quebec in the nineteenth century», Canadian Journal of Economics and Political Science 2 (1957), 195-215.

2 Pierre Camu, Le St-Laurent et les Grands Lacs au temps de la vapeur, 1850-1950 (Montréal : Hurtubise/HMH, 2005), 297.

The Northern Mariner/le marin du nord, XX No. 3, (July 2010), 251-266 


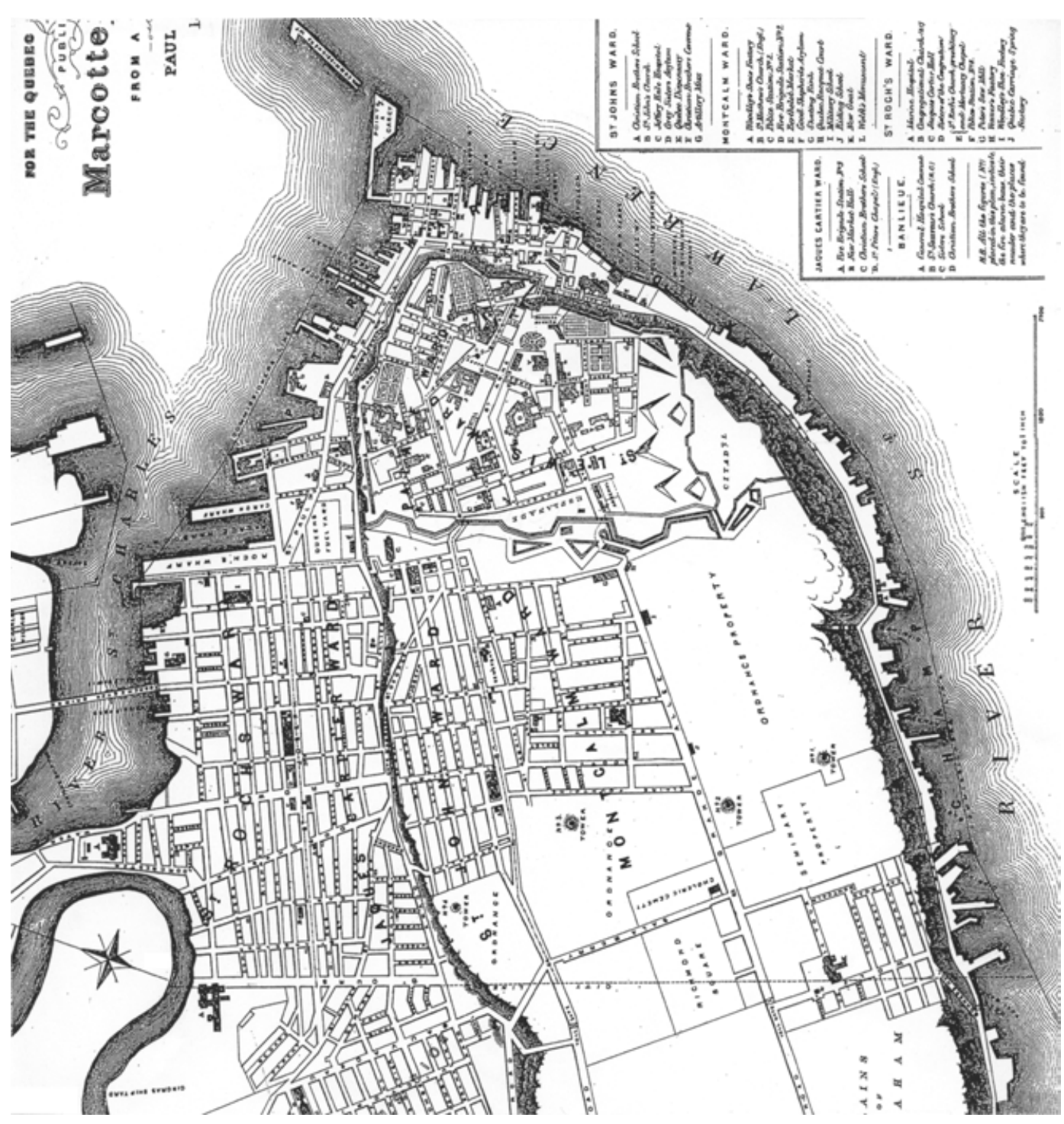

Figure 1: Plan de la cité de Québec pour l'annuaire Québec-Lévis, publié par Marcotte et Levy, d'après un dessin de Paul Cousin, 1871 (Bibliothèque et Archives Canada, NMC 62943.)

des milieux politiques. Un vent de pessimisme soufflait partout. Que faisait les leaders, réagiraient-ils trouveraient-ils des solutions à court et long terme? Ils le firent après des années d'incertitude, de manque de coopération entre les paliers administratifs et, avouons-le, une certaine malchance.

Le mot «déclin »s'applique bien à l'évolution du trafic portuaire océanique seulement, car le trafic intérieur continua et même augmenta. Dans le cas de la construction des grands voiliers, le mot « disparition » est plus approprié, car il s'agit bel et bien de l'élimination d'une activité économique unique et d'une expérience inestimable. 


\section{Déclin du trafic portuaire et ses causes}

Nous présentons l'évolution du trafic portuaire en retenant les trois données statistiques : le nombre de navires entrés et sortis, leur tonnage de jauge et le tonnage des cargaisons manutentionnées sur ses quais. La figure 2 illustre les changements sur un siècle, la période 1851-1951; le tableau 1 montre la moyenne annuelle par période quinquennale entre 1871 et 1900 . Il s'agit dans les deux illustrations du trafic total avec distinctions entre les trafics océanique et intérieur. Le port connut une longue période sombre qui se termina avec la fin de la première guerre mondiale.

\begin{tabular}{|c|c|c|c|c|c|c|}
\hline \multirow[t]{2}{*}{ Période } & \multirow{2}{*}{$\begin{array}{l}\text { Nombre total de } \\
\text { navires entrés et } \\
\text { sortis }\end{array}$} & \multicolumn{2}{|c|}{$\begin{array}{l}\text { Tonnage total des } \\
\text { navires entrés et sortis }\end{array}$} & \multicolumn{3}{|c|}{$\begin{array}{l}\text { Tonnage total des cargaisons } \\
\text { océaniques en tonnes courtes }\end{array}$} \\
\hline & & $\begin{array}{l}\text { Tonnes } \\
\text { courtes }\end{array}$ & $\begin{array}{l}\text { Tonnes } \\
\text { métriques }\end{array}$ & $\begin{array}{l}\text { Tonnage } \\
\text { entré }\end{array}$ & $\begin{array}{l}\text { Tonnage } \\
\text { sorti }\end{array}$ & $\begin{array}{l}\text { Tonnage } \\
\text { total }\end{array}$ \\
\hline $1871-1875$ & 5865 & 1853754 & 1681354 & 102988 & 654395 & 757383 \\
\hline $1876-1880$ & 6969 & 2246957 & 2037989 & 153119 & 880203 & 1033322 \\
\hline 1881-1885 & 6982 & 2078314 & 1885030 & 142802 & 793109 & 935911 \\
\hline $1886-1890$ & 5597 & 2257143 & 2047228 & 104776 & 536862 & 641638 \\
\hline $1891-1895$ & 2290 & 1674336 & 1518622 & 84306 & 444636 & 528942 \\
\hline 1896-1900 & 2151 & 3745887 & 3397519 & 87003 & 485541 & 572544 \\
\hline
\end{tabular}

Tableau 1 - Trafic au port de Québec - Années 1871-1900 - Moyenne annuelle par période quinquennale Source : Tableaux du Commerce et de la Navigation au Canada. Années 1871-1900. Publiés dans les Documents de la Session. Note: Le nombre et le tonnage total des navires incluent les deux grandes catégories de trafic : l'océanique et l'intérieur.

\section{Le trafic océanique}

La diminution du trafic océanique affecta la vie du port et ternit sa réputation au Canada et à l'étranger. Il faudra attendre après la première guerre mondiale pour déceler une reprise et des accroissements de tonnages. Le nombre total de navires océaniques diminua des deux tiers entre 1871-1875 et 1896-1900; par contre, le tonnage de jauge des navires ne diminua que d'un quart; les navires qui continuèrent à s'arrêter dans le port étaient de plus en plus gros à chaque décennie et transportaient davantage de cargaisons. ${ }^{3}$ La donnée la plus significative était celle du tonnage des cargaisons manutentionnées sur les quais. Il y eut, en l'espace de 30 ans, une diminution d'environ 25 pour cent du tonnage total. Si l'on compare cependant avec la période de 1876-1880, la perte est plus significative, elle s'élève à 45 pour cent. ${ }^{4}$

Il n'y a pas eu d'abandon total, on continua d'importer et d'exporter par le port; certains navires faisaient escale à Québec, en route vers ou revenant de Montréal, pour

3 Voici en résumé les données statistiques du trafic océanique seulement:

Nombre de navires Tonnage de jauge

1871-1875 $\quad 1865$

1453754

1896-1900 599

1078166

Ibid., tableaux 8-1 et 8-2, pp. 300 et 305 .

4 Tableau 1. 
PORT DE QUEBEC

A) NOMBRE DE NAVIRES

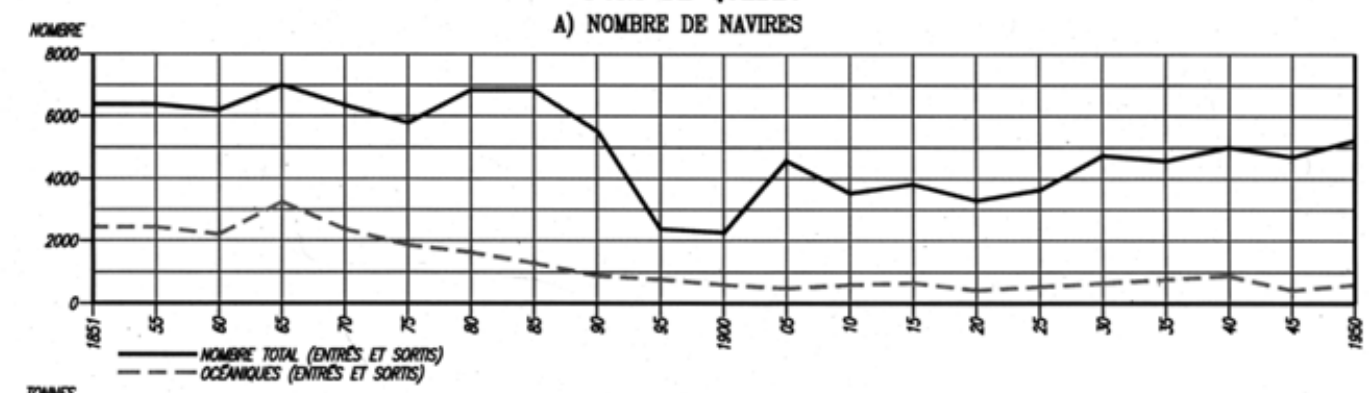

B) TONNAGE DES NAVIRES

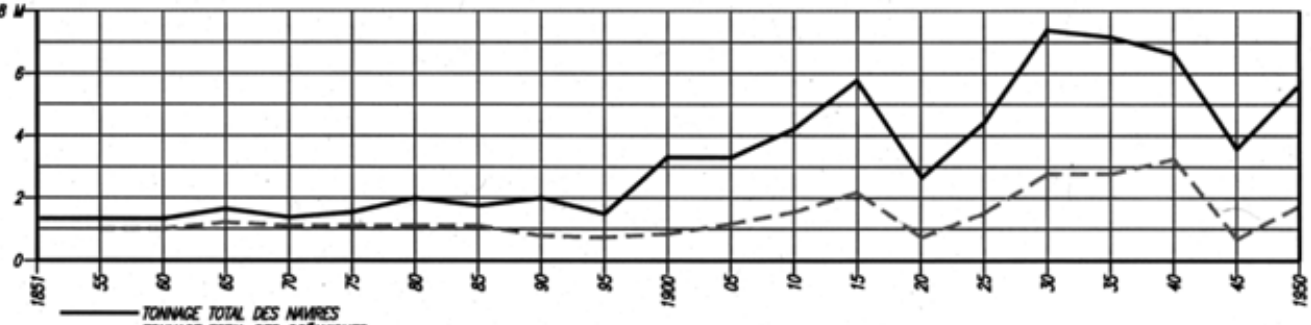

- - mownce row does navers

Irowes

C) TONNAGE DES CARGAISONS

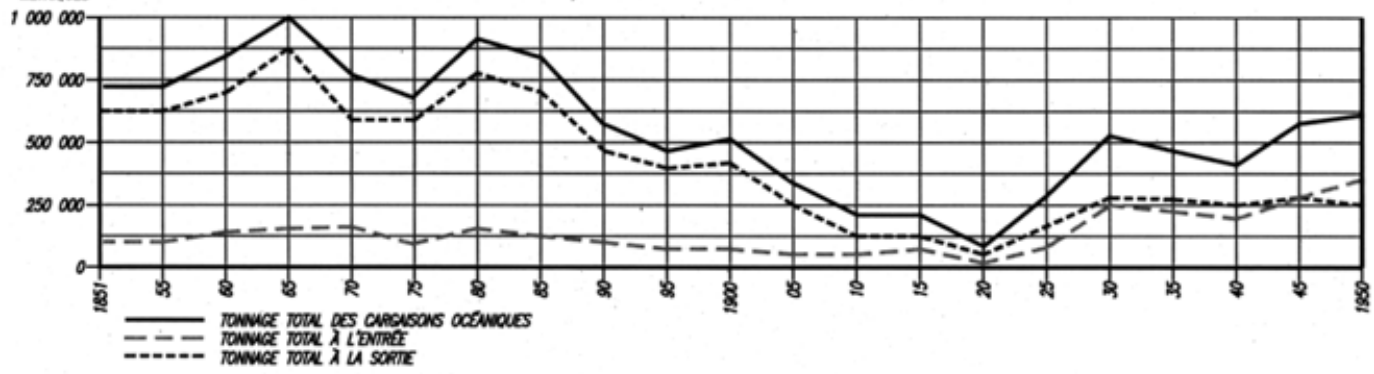

Figure 2: Trafic au port de Québec, période 1851-1950

A) nombre de navires entrés et sortis - nombre total et nombre d'océaniques,

B) tonnage total des navires et des océaniques entrés et sortis,

C) tonnage des cargaisons entrées et sorties.

charger ou décharger. Les pires années, celles où le port enregistra les quantités les plus faibles, survinrent au cours du premier conflit mondial. Ce fut la période quinquennale 19161920, avec comme moyenne annuelle, 276 navires d'un tonnage de 934199 et un tonnage des cargaisons de 146 000. C'était la guerre, le trafic transatlantique était perturbé. Québec était devenu l'avant-port océanique de Montréal dont le tonnage total des cargaisons océaniques passa de 457822 t. en 1871-1875 à 2015676 en 1896-1900. ${ }^{5}$ La catégorie la plus importante à l'exportation était celle des produits forestiers dont la nature changea toutefois avec l'augmentation du papier journal et de la pâte de bois. Les importations de charbon l'emportaient sur tous les autres types de cargaisons déchargées dans le port.

5 Camu, Le St-Laurent et les Grands Lacs au temps de la vapeur, tableaux 8-1 à 8-3 et tableau 8-13. 


\section{Le trafic intérieur ou domestique}

Il y avait deux types de trafic intérieur, celui transporté par goélettes entre les ports des régions de l'estuaire et du fleuve jusqu'à Portneuf et celui transporté par des navires plus gros qui assuraient les liaisons avec les ports du golfe et les canaliers à moteur qui se déplaçaient entre les ports des Grands Lacs et du fleuve. Les goélettes dominaient en aval de Québec, les canaliers l'emportaient en amont. ${ }^{6}$

Le trafic des goélettes diminua avec le temps à cause de la rivalité croissante des chemins de fer exploités toute l'année. Les goélettes à voile furent remplacées par des petits caboteurs à vapeur qui se spécialisaient dans le transport du bois de pâte tandis que les canaliers transportaient du charbon, des céréales et autres produits en vrac.

Dans un tel contexte, le nombre de navires domestiques entre les deux mêmes périodes, 1871-1875 et 1896-1900, diminua de 50 pour cent, mais le tonnage de jauge doubla. Les données statistiques du trafic des cargaisons domestiques est difficile à estimer, il y a très peu de compilations disponibles. La composition du trafic intérieur changea; le charbon, les céréales et le bois de pâte dominaient toutes les autres catégories de produits. Le rôle de port régional de tout l'Est du Québec continua, celui de port de déchargement de matières premières s'accentua. On assistait plus à un changement de types de cargaisons qu'à un déclin de ce trafic.

L'activité portuaire avait diminué, mais pas disparu; en réalité le mot « déclin » réfère à la diminution sévère du trafic océanique, le plus spectaculaire et le plus visible et, jusqu'à un certain point, le plus prestigieux. Le trafic intérieur, plus important que l'autre, moins remarqué, ne connut pas de diminution drastique. On en parlait moins, c'était comme un acquis.

Montréal devint le port terminus de la voie maritime du St-Laurent mais aussi deux autres ports s'affirmaient d'année en année, devenant des ports régionaux intermédiaires, situés entre Québec et Montréal, les ports de Trois-Rivières et de Sorel.

\section{Les causes du déclin du trafic océanique}

En améliorant la voie navigable en amont, vers Montréal, on invitait les navires à remonter le fleuve et pénétrer plus loin vers l'intérieur du continent nord-américain et à servir un marché et un arrière-pays plus étendu, plus riche et plus diversifié que celui du port de Québec. Il y avait une autre possibilité, celle de trouver un fret de retour plus volumineux et plus payant.

Les entrepreneurs et hommes d'affaires montréalais dépensaient beaucoup d'énergie à développer leur port, ils s'activaient constamment à promouvoir leurs intérêts. L'un des objectifs était celui de draguer et d'aménager un chenal plus profond, plus large et plus sécuritaire. Le dragage du fleuve fut une opération quasi constante pendant près d'un siècle. Dès qu'on terminait ou achevait un projet, on passait au suivant. On atteignit une profondeur de 6 mètres entre les deux villes portuaires en 1865; on creusa davantage et l'on atteignit une profondeur de 6,7 mètres en 1878; de 7,6 mètres en 1882 et de 8,2 mètres en 1888 (profondeur actuelle de la voie maritime canalisée, en

6 Ibid., 156-157. Le canalier était un navire à vapeur, type Grands Lacs, construit pour utiliser les canaux du St-Laurent en amont de Montréal. 
amont de Montréal en 1959).

La largeur du chenal en ligne droite et dans les courbes apparaît dans le tableau 2. Les types de navires et leur tonnage de jauge s'adaptèrent constamment afin de profiter des caractéristiques plus avantageuses d'une décennie à l'autre. Ainsi les océaniques furent attirés en amont, faisant de Montréal le port terminus de la navigation transatlantique, situé à plus de $1586 \mathrm{~km}$ à l'intérieur du continent nord-américain. ${ }^{7}$ Avantage indiscutable.

On améliora la voie navigable d'une autre façon depuis l'entrée dans l'estuaire en remontant jusqu'à Montréal; ce fut la multiplicité des aides à la navigation, travaux complémentaires et indispensables. On construisit des phares, on posa des bouées, des balises, des cornes de brume et, dès que ce fut possible, on ajouta des lumières électriques. On améliora la qualité des cartes hydrographiques et on créa un service des signaux avec l'arrivée de la télégraphie. Quelques données statistiques à 25 ans d'intervalle illustrent les améliorations que l'on apporta.

\begin{tabular}{|l|c|c|c|}
\hline $\begin{array}{l}\text { Nombre d'aides à la navigation dans les secteurs de l'estuaire et du fleuve en 1850, } \\
1875 \text { et } 1900\end{array}$ & 1850 & 1875 & 1900 \\
\hline & 29 & 119 & 172 \\
\hline Phares & 2 & 8 & 7 \\
\hline Bateaux-phares & 6 & 16 & 13 \\
\hline Signaux de brume & 149 & 67 & 549 \\
\hline Bouées & --- & 55 & 60 \\
\hline Balises & & 5 & \\
\hline
\end{tabular}

Source : Camu, Le St-Laurent et les Grands Lacs au temps de la vapeur, 77, tableau 2-1.

Les autres causes du déclin du port sont économiques et commerciales. Nous avons retenu deux causes seulement, la première, l'une des plus significatives, était le nombre total d'habitants dans la région immédiate, ce que nous appelons le poids démographique de la région de Québec comparé à Montréal et, la deuxième, les changements profonds des marchés internationaux dans la deuxième moitié du XIXe siècle.

Le nombre et la qualité des personnes d'une ville et de son port sont intimement liés à quatre activités de base : l'industrie manufacturière, le commerce, les services et les moyens de transport. Le nombre total de personnes dans chacune des deux villes portuaires, aux recensements de 1871 à 1901, montre bien l'accroissement rapide de Montréal qui voit sa population doubler en 20 ans, entre 1871 et 1891, et augmenter encore de près de 50000 entre 1891 et 1901 . Québec, pendant ce temps-là, faisait du « sur place », moins de 10000 personnes de plus en trente ans (de 1871 à 1901). En

7 Distance entre le détroit de Belle-Isle et l'entrée du canal Lachine. Les hommes d'affaires, les entrepreneurs, les armateurs et autres personnes du monde maritime de Montréal firent du projet de dragage, de l'aménagement du port et du raccordement aux réseaux nationaux ferroviaires leurs priorités dans la deuxième moitié du XIXe siècle. Cela faisait partie de la rivalité entre Montréal et Québec. K.S. Mackenzie, "The Montreal-Quebec Rivalry », Seaports and the Shipping World (July 1985), 36-8 et 69. 


\begin{tabular}{|c|c|c|c|c|c|c|c|c|c|c|}
\hline \multicolumn{2}{|c|}{$\begin{array}{l}\text { Projet de dragage } \\
\text { profondeur }\end{array}$} & \multicolumn{2}{|c|}{$\begin{array}{l}\text { Distance } \\
\text { approximative à } \\
\text { draguer }\end{array}$} & \multirow{2}{*}{$\begin{array}{l}\text { Début des } \\
\text { travaux } \\
\text { Année app } 1\end{array}$} & \multirow{2}{*}{$\begin{array}{l}\text { Fin des } \\
\text { travaux } \\
\text { roximative }\end{array}$} & \multicolumn{2}{|c|}{$\begin{array}{l}\text { Largeur du } \\
\text { chenal en ligne } \\
\text { droite }\end{array}$} & \multicolumn{2}{|c|}{$\begin{array}{l}\text { Largeur du chenal } \\
\text { dans les courbes }\end{array}$} & \multirow[t]{2}{*}{ Responsabilités } \\
\hline Mètres & Pieds & $\mathrm{Km}$ & Miles & & & Mètres & Pieds & Mètres & Pieds & \\
\hline \multicolumn{11}{|c|}{ SECTEUR QUÉBEC-MONTRÉAL } \\
\hline 4,8 & 16 & 24 & 15 & 1851 & 1854 & 76/91 & $250 / 300$ & 91 & 300 & $\begin{array}{l}\text { Commissaires, } \\
\text { Port de } \\
\text { Montréal }\end{array}$ \\
\hline 6,0 & 20 & 48 & 30 & 1855 & 1865 & 91 & 300 & 91 & 300 & $\begin{array}{l}\text { Commissaires, } \\
\text { Port de } \\
\text { Montréal }\end{array}$ \\
\hline 6,7 & 22 & - & - & 1873 & 1878 & 91 & 300 & $91 / 121$ & $300 / 488$ & $\begin{array}{l}\text { Commissaires, } \\
\text { Port de } \\
\text { Montréal }\end{array}$ \\
\hline 7,6 & 25 & 80 & 50 & 1879 & 1882 & 99 & 325 & $91 / 129$ & $300 / 425$ & $\begin{array}{l}\text { Commissaires, } \\
\text { Port de } \\
\text { Montréal }\end{array}$ \\
\hline 8,2 & 27,5 & 80 & 50 & 1883 & 1888 & 106 & 350 & $137 / 152$ & $450 / 500$ & $\begin{array}{l}\text { Commissaires, } \\
\text { Port de } \\
\text { Montréal }\end{array}$ \\
\hline 9,1 & 30 & 160 & 100 & 1899 & $1907 / 1915$ & 121 & 400 & $152 / 228$ & $500 / 750$ & $\begin{array}{l}\text { M.T.P. (1889- } \\
\text { 1904) et } \\
\text { M.M.P. (1904- } \\
\text { 1936) }\end{array}$ \\
\hline $10,6^{*}$ & 35 & 160 & 100 & 1910 & 1950 & 137 & 450 & $152 / 243$ & $500 / 800$ & $\begin{array}{l}\text { M.M.P. (1910- } \\
1936)\end{array}$ \\
\hline $10,6^{* *}$ & 35 & 160 & 100 & 1936 & 1952 & 243 & 800 & 243 & 800 & $\begin{array}{l}\text { M.T. (1936- } \\
1950)\end{array}$ \\
\hline \multicolumn{11}{|c|}{ SECTEUR QUÉBEC-LA TRAVERSE (EN AVAL) } \\
\hline 9,1 & 30 & 12,2 & 7,6 & 1907 & 1912 & 304 & 1000 & 304 & 1000 & $\begin{array}{l}\text { M.M.P. (1904- } \\
1936)\end{array}$ \\
\hline 10,6 & 35 & 20,9 & 13 & $19 \ldots$ & 1950 & 304 & 1000 & 304 & 1000 & $\begin{array}{l}\text { M.T. (1936- } \\
1950)\end{array}$ \\
\hline \multicolumn{11}{|c|}{ SECTEUR RIVIÈRE SAGUENAY (BAGOTVILLE-CHICOUTIMI) } \\
\hline 6 & 20 & 14,4 & 9 & & & 76 & 250 & 76 & 250 & \\
\hline
\end{tabular}

Tableau 2 - Dragage du fleuve Saint-Laurent, 1851-1950 Sources : Rapport annuel du ministère des Travaux publics, 1867 à 1904; Rapport annuel du ministère de la Marine et des pêcheries, 1904 à 1936; N. Corley, The St Lawrence Ship Channel, 1805-1865; H.L. Land, The Development of the Saint-Lawrence Ship Channel. Notes : M.T.P. : Ministère des Travaux publics, M.M.P. : Ministère de la Marine et des Pêcheries, M.T. : Ministère des transports.

*Étiage de 1897, **Étiage de 1934 
1871, la population de Montréal était le double de celle de Québec, en 1901, trente ans plus tard, il y avait quatre fois plus de personnes à Montréal qu'à Québec. Montréal progressait à vive allure, Québec stagnait, elle ne faisait pas le poids.

\begin{tabular}{|l|l|l|l|l|}
\hline $\begin{array}{l}\text { Année de } \\
\text { recensement }\end{array}$ & $\begin{array}{l}\text { Montréal } \\
\text { Cité }\end{array}$ & $\begin{array}{l}\text { Québec } \\
\text { Cité }\end{array}$ & $\begin{array}{l}\text { Montréal } \\
\text { Région }\end{array}$ & $\begin{array}{l}\text { Québec } \\
\text { Région }\end{array}$ \\
\hline 1871 & 107225 & 59699 & 126314 & 63390 \\
\hline 1881 & 140747 & 62446 & 170745 & 71599 \\
\hline 1891 & 216650 & 63090 & 250165 & 73942 \\
\hline 1901 & 267730 & 68840 & 324880 & 80039 \\
\hline
\end{tabular}

Source: recensement du Canada 1901, vol. I, p. 22 et Linteau, Durocher, Robert, Histoire du Québec Contemporain, tome 1, 170, tableau 4. Note: La région de Québec inclut Lévis et Lauzon; celle de Montréal inclut la banlieue immédiate.

L'Angleterre annulait ses lois protectrices que furent les Navigations Laws et les Corn Laws entre 1840 et 1850 et abandonnait le mercantilisme qui l'avait bien servie jusqu'ici. Le Canada ne jouissait plus de tarifs spéciaux, douaniers, de transport ou d'exportation. Le marché privilégié de Grande-Bretagne s'ouvrait au grand commerce international. L'Angleterre, qui était devenue l'entrepôt et le siège social de son empire, souhaitait élargir son rôle à l'échelle mondiale. Il y eut réorientation de ses marchés et réorganisation de ses postes et comptoirs qui affectèrent son trafic triangulaire transatlantique dans lequel le Canada jouait un rôle majeur et le port de Québec une fonction nationale. Le marché protégé de Grande-Bretagne cessa et les exportations de bois diminuèrent. Heureusement que le trafic des céréales, après 1900, augmenta rapidement et compensa la perte partielle de son trafic océanique.

Un autre marché potentiel prenait de plus en plus d'ampleur. Il était plus rapproché, c'était le marché américain, accessible non seulement par eau mais aussi par rail avec la construction effrénée des chemins de fer dans la deuxième moitié du XIXe siècle. Les Etats-Unis remplaçaient graduellement la Grande-Bretagne.

Les Etats-Unis et le Canada participèrent presqu'en même temps à la conquête des Prairies, de l'Ouest et au développement spectaculaire des territoires qui longeaient le Pacifique. Il y eut le développement des grands espaces céréaliers des Prairies, l'immigration massive vers ces nouveaux territoires, la naissance du Midwest américain, de villes et cités et le tout relié par un réseau ferroviaire qui s'ajoutait et prolongeait le réseau de voies canalisées des Grands Lacs, du Mississippi et de l'Ohio. Le port et la ville de Québec se trouvaient marginalisés dans le coin nord-est du continent nord-américain tandis que Montréal en profitait pleinement avec son réseau de voies navigables (l'Outaouais, le Richelieu et le Saint-Laurent en amont) et le développement rapide de voies ferrées dans toutes les directions qui raccordaient le port aux réseaux nationaux.

L'évolution des arrière-pays nord-américains favorisa une économie continentale au détriment d'une économie maritime. Le port et la ville de Québec étaient situés en marge des grands courants de circulation et perdirent au change. ${ }^{8}$

8 Lemelin a bien analysé et décrit les causes commerciales et économiques du déclin du trafic océanique dans son article déjà cité. 


\section{Les changements technologiques et l'effondrement de la construction navale}

L'autre déclin, comme l'a bien décrit Albert Faucher, arriva en même temps dans la deuxième moitié du XIXe siècle. Il fut plus néfaste, entrainant la disparition des chantiers maritimes où l'on construisait des navires en bois. Un seul chantier survivra.

Deux changements technologiques révolutionnèrent l'exploitation des navires : celui du remplacement de la voile par la vapeur, et celui du remplacement des coques en bois par des coques en fer d'abord, et en acier ensuite.

L'utilisation de la vapeur au lieu de la voile pour propulser les navires les rendaient indépendants des vents qu'on avait utilisés depuis des millénaires et des autres conditions climatiques, jusqu'à un certain point. Pour remonter le fleuve, on n'attendait plus pendant des semaines que le vent souffle du nord-est. On établit des horaires de départ à date fixe, une régularité dans la rotation des navires et l'implantation de lignes maritimes qui avaient tout avantage à relier les ports les plus actifs et offraient un bon fret de retour. Le remplacement des grandes roues à aubes par des hélices améliora considérablement la propulsion des navires. Avec la révolution industrielle, d'autres applications suivirent, comme le fonctionnement à la vapeur de treuils, cabestans et grues qui accélérèrent l'arrimage et la manutention des cargaisons.

L'utilisation grandissante des coques en fer et en acier eut un effet désastreux sur l'économie industrielle de la région métropolitaine et sa vie portuaire. On peut parler de disparition, ce fut la fin de la construction des grands voiliers en bois, une industrie qui existait déjà au temps de la Nouvelle-France et qui avait redémarré vers 1780-1785 au temps du régime britannique. Cette industrie connut son apogée avec le lancement des trois-mâts de type « carré » et « barque » entre 1845 et 1870. On les appelait les « timber ships ».

Eileen Reid Marcil a compilé une liste complète des grands voiliers de commerce construits dans une trentaine de chantiers maritimes du port de Québec, entre 1763 et $1893 .{ }^{9}$ La réputation des «timber ships », les grands trois-mâts à voile construits et lancés à Québec et ailleurs le long du fleuve et de l'estuaire, s'étendait aux milieux maritimes internationaux du XIXe siècle. On a construit dans la trentaine de chantiers du port plus de 800 navires de ce type d'un tonnage total de 625000 tonneaux entre 1850 et $1893 .{ }^{10}$ On employait chaque année entre 2100 et 2800 ouvriers. ${ }^{11}$ C'était une industrie majeure dans la région et le port de Québec. Elle ne s'adapta pas à la construction des coques en fer et en acier, à la technologie de la sidérurgie et de la métallurgie et au savoirfaire nouveau. Un seul grand chantier survivra, ce sera le chantier Davie à Lévis en face de Québec. ${ }^{12}$

9 Eileen Reid Marcil, The Charley-Man : A History of Wooden Shipbuilding at Quebec, 17631893 (Kingston : Quarry Press, 1995), appendices B et C.

10 Ibid., Appendice B. Liste des voiliers de 100 tonneaux et plus.

11 Ibid., 97, 154, 204 et appendice D. C'est la période 1851-1871.

12 Eileen Reid Marcil, Tall Ships and Tankers : The History of the Davie Shipbuilders (Toronto: McClelland and Stewart, 1997). Le chantier Davie survécut, il passa de la construction de coques en bois à des coques de fer et en acier. 
Les chantiers étaient dispersés dans les anses le long du fleuve, en amont du quai de la traverse Québec-Lévis ainsi que le long de la rive droite du fleuve et de la rivière StCharles. Avec la fin de cette industrie et la diminution des exportations de bois, le port disposait de nombreux sites à réaménager et à développer.

\section{La prise en main de l'expansion portuaire}

La Commission du Havre de Québec fut fondée en 1858. Avant cette date, l'administration et la gestion du port relevait de la Maison de la Trinité qui assumait aussi le contrôle de la navigation du St-Laurent depuis l'entrée dans le golfe jusqu'à Portneuf en amont. ${ }^{13}$ C'était la première fois qu'un groupe de personnes se dévouaient exclusivement aux affaires maritimes du port et de son arrière-pays. Avant de planifier et de songer à son développement et à son expansion, on se devait de connaître et d'apprécier ce qu'était le port.

Puisque tous les quais et autres installations dans les anses voisines appartenaient au secteur privé, on procéda pendant la première décennie à l'acquisition de terrains susceptibles de contribuer à une administration plus rationnelle et à un développement contrôlé en vue d'une expansion possible. La politique d'achats de terrains contribua, avec le temps, à créer une entité homogène qui s'appellera le port de Québec.

« Dans les premières années, la Commission construisit le quai de la Pointe-àCarcy, y érigea des hangars, acheta quelques quais avoisinants, uniformisa la profondeur d'eau au pied des quais privés et aménagea un brise-lames à l'embouchure de la rivière Saint-Charles ». ${ }^{14}$ Malheureusement, tout cela coûtait cher, la Commission devait beaucoup d'argent. Les créanciers prirent le contrôle du port et arrêtèrent les travaux en 1869. Le gouvernement se sentait responsable de l'arrêt de modernisation du port qui avait joué un si grand rôle pendant plus de 200 ans, il versa à la Commission du port, réorganisée et relancée, une somme de 4 millions de dollars. ${ }^{15}$

Le projet le plus exigeant que l'on favorisa en 1875 fut de construire le Bassin Louise à l'embouchure de la rivière Saint-Charles et de tripler la superficie du territoire portuaire. L'autre projet que préconisait le gouvernement fédéral était la construction d'un bassin de radoub, d'une cale sèche, afin de réparer les navires et conserver dans la région de Québec, une expertise exceptionnelle basée sur la construction des grands voiliers. Les deux projets furent lancés à peu près en même temps, en 1875-1876.

Les plans et devis du Bassin Louise, ainsi nommé en l'honneur de la princesse Louise, fille de la reine Victoria et épouse du marquis de Lorne, gouverneur général du Canada, proposaient un échéancier en trois phases par trois contrats successifs. Le

13 Victoria 23, 1858, chapitre 32.

14 A. Bonneau, «L'histoire du Bassin Louise : un concours de circonstances ", L'Escale no 35, 23-24. Il résume bien la controverse au sujet de l'appel d'offres et des plans de réaménagement.

15 Versement de 4 millions de dollars fait en 1873. Bonneau écrit que cette somme représentait «l'aboutissement de pressions exercées depuis plus de 50 ans » par les gens du milieu maritime et d'affaires de Québec. 


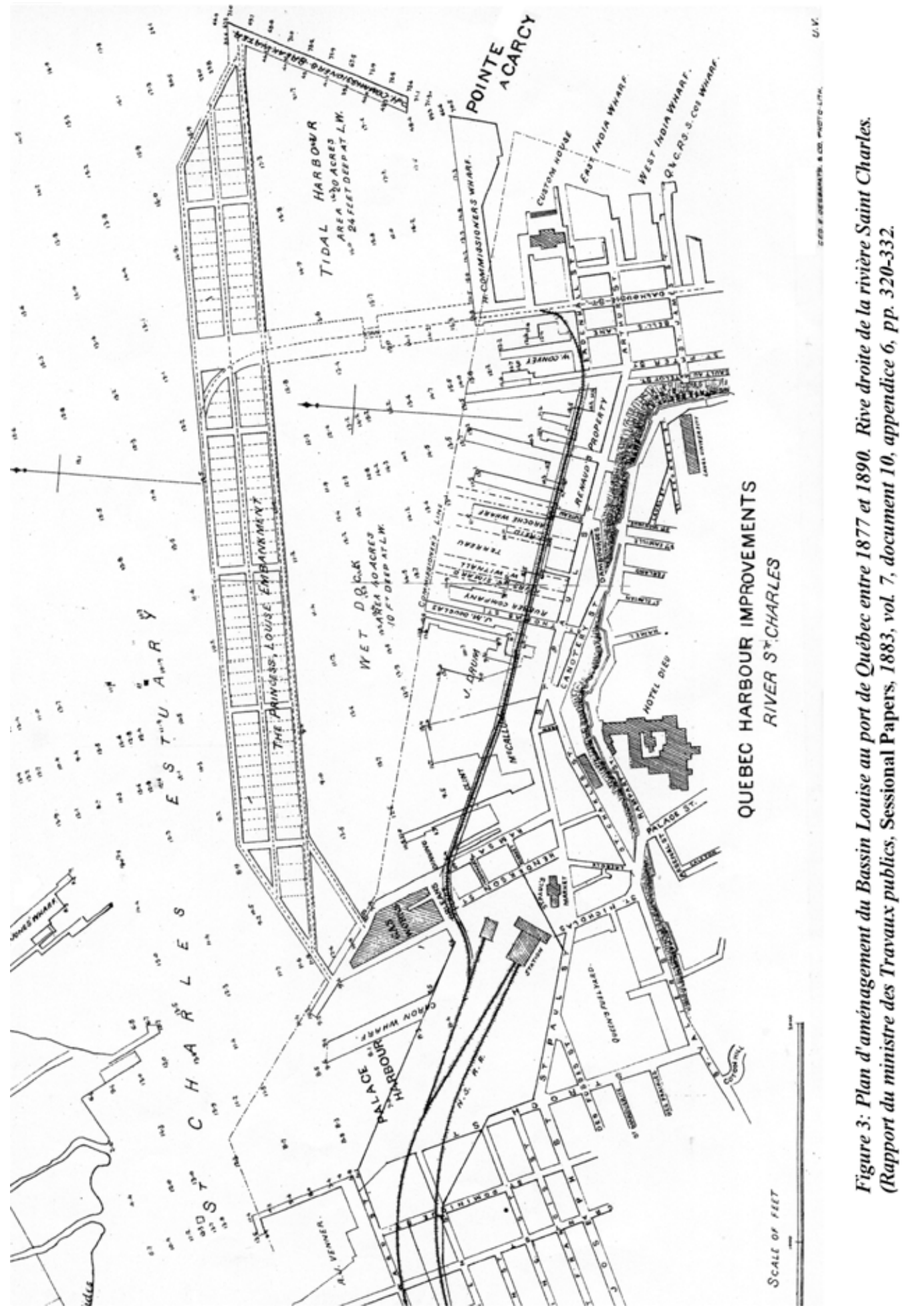




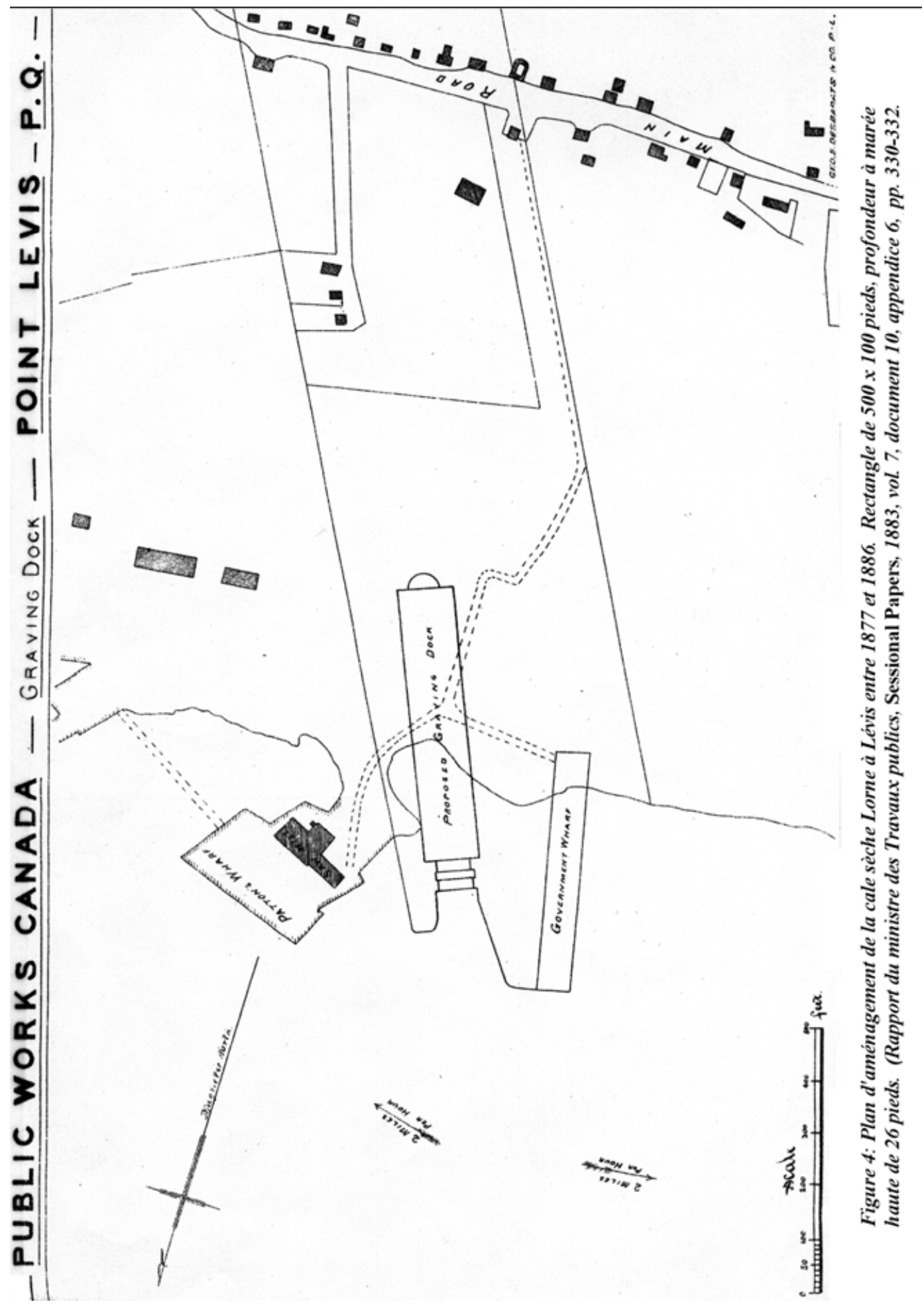


premier contrat, octroyé en 1877, consistait en la construction d'une jetée de $1066 \mathrm{~m}$ de longueur et de $106 \mathrm{~m}$ de largeur, le long de la rive droite de la rivière Saint-Charles. La jetée se rattachait au brise-lames de la Pointe-à-Carcy. Le deuxième contrat concernant la construction «d'un môle perpendiculaire à la jetée, de manière à circonscrire un bassin intérieur (à flot) de quelques 16 hectares d'eau et un bassin extérieur (à marée) qui en fait $8 » .{ }^{16}$ Le rattachement à l'ouest de la jetée au rivage faisait partie du deuxième contrat. Le troisième contrat référait « à la mise en place d'un égout collecteur rendu nécessaire pour éviter que l'eau du bassin à flot ne devienne fétide ». Longeant les quais de la rivière Saint-Charles, « il canalise les nombreuses installations sanitaires qui s'y déversent et se jettent dans le fleuve... ». ${ }^{17}$ Les travaux s'échelonnèrent de 1877 à 1890 , une période de 15 ans; on disposait en 1890 de deux bassins d'une profondeur de 8 mètres, profondeur semblable à la troisième voie maritime du Saint-Laurent inaugurée en 1959 (figure 3).

Le projet de la cale sèche de Lorne à Lévis, nommé d'après le marquis de Lorne, consistait en un rectangle de $152 \mathrm{~m}$ de longueur par $30 \mathrm{~m}$ de largeur et de $7,9 \mathrm{~m}$ de profondeur à marée haute, ce qui convenait largement aux besoins des navires commerciaux de l'époque. Les pompes avaient une capacité de 2271 litres par minute. Les travaux débutèrent en 1877 et se terminèrent en 1886. Le port de Québec-Lévis s'était complètement transformé et modernisé en 15 ans. De rivages d'anses et de quais perpendiculaires au fleuve avec peu d'espace pour la manutention des marchandises en vrac ou empaquetées, on offrait aux armateurs, expéditeurs et commerçants un nouveau port et une cale sèche voisine du chantier maritime de George T. Davie (figure 4). ${ }^{18}$

Ce qui manquait au port de Québec afin de profiter pleinement de ses nouvelles installations, c'étaient des liaisons avec les chemins de fer en pleine expansion au Canada et aux Etats-Unis. L'initiative de relier le port et la ville par rail, aux réseaux nationaux et atteindre des arrière-pays éloignés, vint surtout du secteur privé avec quelques projets du secteur public. On regretta que le raccordement à des réseaux nationaux se fit avec un assez long retard; on relia, par exemple, Montréal à Québec par voie ferrée le long de la rive nord du fleuve en 1879, et on rejoignit les réseaux de chemins de fer de la rive sud par le pont de Québec en 1917. Pierre Dufour écrivit que la ville et le port «avaient manqué le train ». ${ }^{19}$

Les chemins de fer rivalisaient avec les autres modes de transport, par eau ou par route, mais sont aussi complémentaires aux activités portuaires. Ce fut le cas dans l'extension des voies ferrées jusqu'au cœur du port de Québec. Les liaisons par rail aux régions du Lac Saint-Jean-Saguenay, au comté de Charlevoix, à l'Abitibi, aux Prairies canadiennes, à celles de Portneuf et de la Mauricie et, sur la rive sud, avec les régions de Beauce, du Bas-du-Fleuve, de la Gaspésie, de l'Estrie et des Bois-Francs étaient une nécessité (figure 5).

16 A. Bonneau, « Le bassin de la princesse Louise », L'Escale n 37, 15-19

17 Ibid., 18.

18 K. S. Mackenzie, "The Quebec City Graving Dock », Seaports and the Shipping World (January 1987), 36-37 et 56.

19 P. Dufour, Esquisse de l'évolution physique du port de Québec, des origines à 1900, Rapport de recherche ${ }^{\circ} 23$ (Ottawa : Parcs Canada, 1981), 21. 
Il y eut deux développements parallèles de l'arrivée du rail dans la région métropolitaine de Québec-Lévis : le premier et le plus ancien fut celui au sud du SaintLaurent, le deuxième empruntait un parcours assez rapproché de la rive nord. ${ }^{20}$

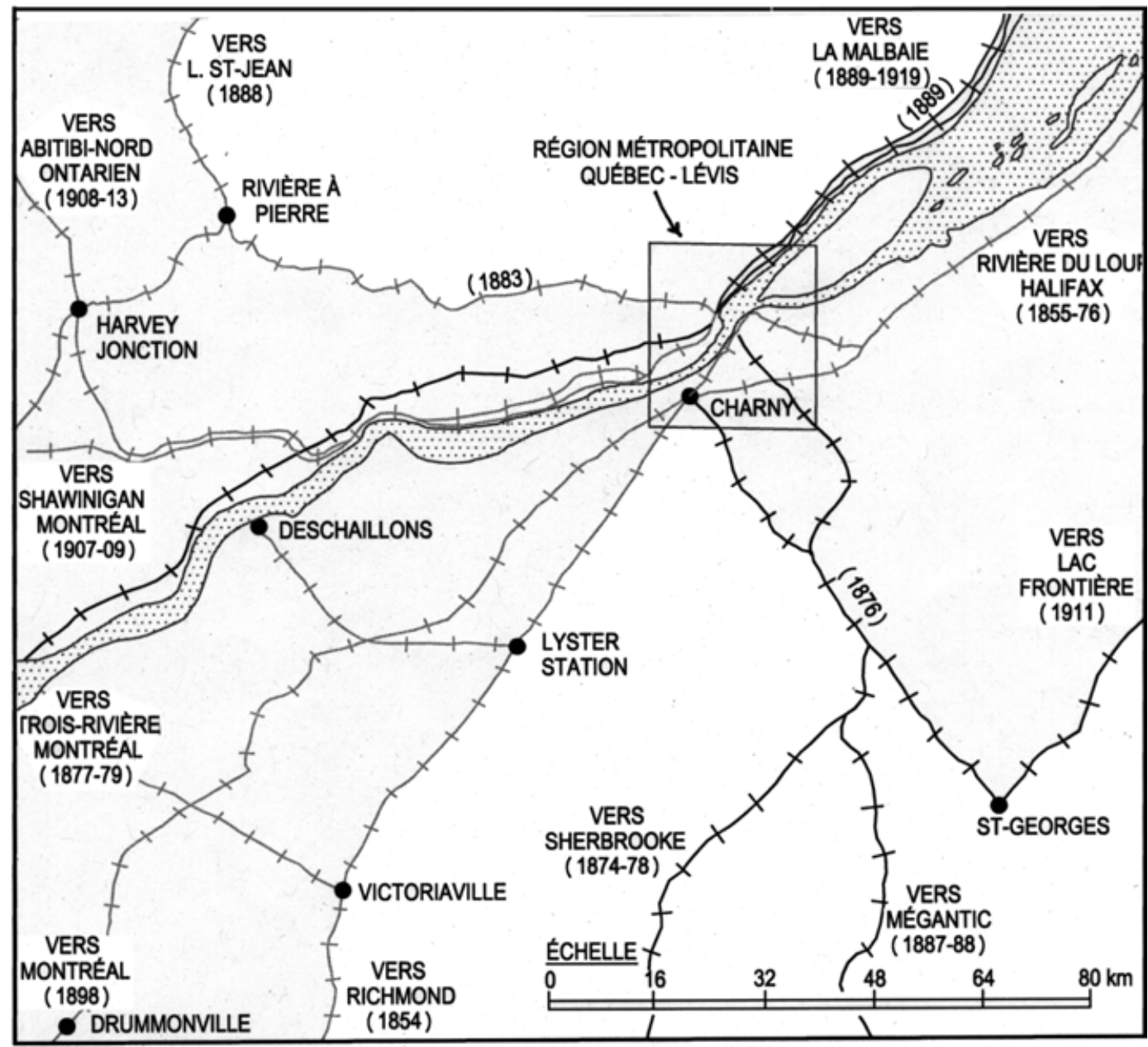

Fig.5 - RÉSEAUX FERROVIAIRES AUTOUR DE LA RÉGION MÉTROPOLITAINE DE QUÉBEC-LÉVIS

( ) Dates du commencement de l'exploitation de la ligne + Chemin de fer

La première ligne construite fut celle au sud du fleuve qui suivit une ligne de Longueuil-St-Lambert à Saint-Hyacinthe, Richmond, Victoriaville et Lévis. Elle fut achevée en 1854; en 1860, on la prolongeait jusqu'à Rivière-du-Loup. En 1876, elle atteignait Halifax, reliant ainsi par voie ferrée les provinces du Nouveau-Brunswick et de la Nouvelle-Écosse aux provinces de Québec et d'Ontario. C'était la ligne de l'Intercolonial. Toujours sur la rive-sud, à partir de Lévis, on relia la vallée de la rivière

20 C. Andreae, Lines of Country: An Atlas of Railways and Waterways History in Canada (Erin, ON: Boston Mills Press, 1997), plates 9-13 et 139. 
Chaudière (la région de la Beauce) entre 1874 et 1876. Durant la décennie des années 1890, on construisit une ligne entre Drummondville et Lévis (1898) qui deviendra la ligne principale entre Montréal et Québec via la rive sud du Saint-Laurent.

On espérait réunir ces lignes à la ville de Québec en 1907. Malheureusement une partie du pont de Québec s'effondra une première fois, cette année-là, et une deuxième fois, quand la partie centrale du pont de type cantilever s'affaissa en 1916. L'année suivante, en 1917, on reliait enfin les voies ferrées de la région de Québec; ce fut 58 ans après l'ouverture du pont Victoria au trafic ferroviaire de la rive sud dans la région de Montréal.

La première voie ferrée construite sur la rive nord dans la région de Québec fut la petite ligne Québec-Loretteville que l'on inaugure en 1870. Il faudra attendre encore neuf ans pour que la grande ligne Québec-Montréal soit achevée et exploitée par la Compagnie Québec, Montréal, Ottawa et Occidental, reliant les trois villes en passant par le territoire du Québec. Les travaux se terminèrent en 1879, Québec était désormais reliée à Trois-Rivières et à la métropole.

Les politiciens, hommes d'affaires et entrepreneurs de la région de Québec comprenaient bien que leur ville et le port étaient marginalisés par rapport aux nouveaux courants commerciaux et économiques du Canada et des Etats-Unis dans le dernier quart du XIXe siècle. Le chemin de fer Intercolonial reliait Halifax à Montréal et Toronto en 1876, sans passer par Québec même, il longeait le fleuve en passant par Lévis sur la rive sud. Est-ce que la construction du deuxième grand réseau ferroviaire national, le Canadien Pacifique, aboutirait à Québec, rattachant ainsi la région, la ville et son port aux réseaux nationaux et nord-américains?

Après l'achèvement de la voie ferrée Montréal-Vancouver en 1885, que ferait la société ferroviaire du Canadien Pacifique qui souhaitait prolonger sa ligne nationale vers les provinces maritimes et s'étendre de l'Atlantique au Pacifique? Le tracé favorisait la route la plus directe, un pont au dessus du Saint-Laurent près de Lachine, un parcours via les Cantons de l'Est, l'État du Maine et l'arrivée au port de Saint-Jean au NouveauBrunswick. Un tel parcours favorisait encore une fois Montréal au détriment de Québec. «Le projet de la "Short Line» par Sherbrooke...laissait intacte la prospérité de Montréal, et même pourrait la consolider, mais consacrait la stagnation de Québec et de son district, en détournant le commerce de l'Ouest $\gg .^{21}$

Les gens de Québec répliquèrent par une série de résolutions présentées dans un mémoire au Parlement par ses députés : ils voulaient que le Canadien Pacifique ait son terminus d'été à Québec, que sa ligne nationale soit construite en territoire canadien, que l'on construise un pont à Québec entre les rives nord et sud du fleuve, que l'on relie son réseau avec l'Intercolonial à Lévis et que l'on intègre au réseau la ligne de la Rive Nord entre Montréal, Trois-Rivières et Québec, propriété de la Compagnie du Grand Tronc et auparavant, propriété de la Compagnie Québec, Montréal, Ottawa et Occidental. ${ }^{22}$ Le

21 Robert Rumilly, Histoire de la Province de Québec, vol. IV (Montréal : Éditions Valiquette, 1940), 220

22 Cette société avait appartenu au gouvernement de la Province de Québec dont le directeur 
Canadien Pacifique acheta la ligne de la Rive Nord du Grand Tronc en 1885; cependant, la compagnie paracheva la ligne du sud qui passait par l'État du Maine, le trafic transcontinental échappait encore une fois au port de Québec. ${ }^{23}$

La ligne vers Loretteville, dans les années qui suivirent, fut prolongée jusqu'à Rivière-à-Pierre en 1883 et, de là, se séparait en deux lignes très importantes pour l'avenir du port et de la ville. La ligne vers le nord-ouest, longue de milliers de kilomètres fut complétée en 1910. Elle reliait Québec à la région de l'Abitibi, au nord de l'Ontario et atteignait Winnipeg et les grands territoires des Prairies. Cette ligne transcontinentale fut administrée par la compagnie Canadien Northern, puis la compagnie du Grand Tronc et, finalement, par le Canadien-National. On transporta des milliers de boisseaux de céréales pendant des décennies, surtout en hiver, vers les élévateurs et silos à céréales du port de Québec. On construisit un silo d'une capacité d'un million de boisseaux sur la jetée du Bassin Louise en 1900; le site remplit toujours la même fonction. La deuxième ligne, celle vers le nord-est, fut construite au cours des mêmes années, elle atteignit Chambord et la région du Lac Saint-Jean en 1888 et Chicoutimi et le pays du Saguenay en 18921893.

Le Grand Tronc atteignit Québec en 1907 en prolongeant sa ligne de MontréalShawinigan et Sainte-Foy afin de raccorder cette ligne par le nouveau pont de Québec en construction. Il faudra attendre une autre décennie, en 1917, pour relier les deux réseaux. On prolongea cette ligne entre Sainte-Foy et le Marché Champlain (site du traversier actuel) en 1915. On ouvrit une voie ferrée le long du fleuve sur la rive nord en 1889 entre Québec et Sainte-Anne-de-Beaupré, lieu de pèlerinage célèbre. On prolongea cette ligne vers Baie St-Paul et La Malbaie en 1917-1919.

Québec disposait alors de voies ferrées qui s'allongeaient tout autour, avec un retard sur Montréal qui varia entre 30 et 60 ans. Même si l'on considère le rail comme le grand rival des transports par eau dans le bassin Saint-Laurent Grands Lacs, il n'en demeure pas moins que le réseau ferroviaire agissait aussi comme complémentaire aux infrastructures portuaires et aux lignes maritimes.

\section{Conclusion}

Le port de Québec en 1900 était méconnaissable. Il s'était modernisé et était prêt à répondre aux attentes des armateurs et de leurs clients. Quelques hommes d'affaires, politiciens et entrepreneurs ont cru dans son avenir au cours des années sombres de son déclin. Ce furent J. Gibb Ross, Henry Fry, R.R. Dobell, la famille Price et les maires Francois Langelier et Pierre Garneau. Ils ne connurent pas la reprise du trafic et de ses activités qui s'amorça après la première guerre mondiale et se confirma après la grande dépression des années 1930.

général fut L.A. Sénécal pendant quelques années. Rumilly a consacré des pages à cette question dans son vol. IV.

23 Le Canadien Pacifique avait acheté la section ouest, la ligne Ottawa-Montréal et ses embranchements, en 1881-1882. 